\title{
Slowing too slowly: The same message in a different bottle, or an opportunity to make lung surgery safer?
}

Daniel J. Boffa, MD

See related article on pages 1168-73.

The observation by $\mathrm{Ha}$ and colleagues ${ }^{1}$ that patients who take longer to slow their (presumably elevated) heart rates after a 6-minute walk are more likely to experience complications after lung cancer surgery, could represent an exciting new direction for risk management in this population. The specific metric (ie, failure to decrease heart rate by at least $13 \mathrm{bpm} 60$ seconds after completing a 6-minute walk) is relatively inexpensive and simple. Therefore impaired heart rate recovery has great appeal for risk assessment across a wide array of practice environments. The risk-adjusted correlation between impaired heart rate recovery and complications is impressive (odds ratio, 4.9); however, the study did include a relatively small $(\mathrm{n}=96)$, highly selected subset (only $16 \%$ of surgical lung cancer patients during the study time period were assessed). The relationship between impaired heart rate recovery and complication risk will need to be better characterized (eg, inclusion of surgical approach in the model and teasing out the high risk values from continuous pulmonary functions). The practical aspects of the test will need to be clarified, such as the relationship between maximal heart rate and test results (it is curious that beta-blocker use and history of atrial dysrhythmia did not seem to effect results) and the reproducibility of the test results (ie, whether or not a patient would fall into the same recovery category every time the test was performed). Finally, the clinical relevance of the risk determination is unclear because the complications studied included a spectrum of nonlethal complication severity that may or may not have long-term importance. This is obviously critical to establish, because some clinicians may interpret elevated risk for nonlethal short-term adversity as being prohibitive, when in fact it has no bearing on long-term outcome.

Interestingly, the greatest potential for heart rate recovery testing may be in the ability of the test to be followed. More specifically, if the test result is a reflection of cardiovascular fitness, then heart rate recovery should be modifiable as the patient gets into better (or worse) shape. Clinicians could conceivably use this metric to gauge patient optimization for surgery. Even if the heart rate recovery metric was only found to be predictive of short-term events (ie, no long-term relevance) the test could have tremendous financial implications (eg, length of stay and cost). One could easily envision a randomized trial in which patients with impaired heart rate recovery were sent for rehabilitation for 3 weeks, and the 6-minute walk test repeated, to see if the complication risk could be normalized within this high-risk cohort. Therefore this line of study not only represents a novel way of obtaining currently available information (ie, patient risk for complications), but the impaired heart rate recovery may offer a real-time glimpse at patient fitness (and efforts to improve it) that could potentially be used to make surgery safer.

\section{Reference}

1. Ha D, Choi H, Zell K, Raymond DP, Stephans K, Wang XF, et al. Association of impaired heart rate recovery with cardiopulmonary complications after lung cancer resection surgery. J Thorac Cardiovasc Surg. 2015;149:1168-73.

\footnotetext{
From the Department of Thoracic and Cardiovascular Surgery, Yale University, New Haven, Conn.

Disclosures: Author has nothing to disclose with regard to commercial support.

Received for publication Dec 19, 2014; accepted for publication Dec 19, 2014; available ahead of print Jan 31, 2015.

Address for reprints: Daniel J. Boffa, MD, Department of Thoracic and Cardiovascular Surgery, Yale University, 9500 Euclid Ave, H35, New Haven, CT 06250-8062 (E-mail: daniel.boffa@yale.edu).

J Thorac Cardiovasc Surg 2015;149:1174

0022-5223/\$36.00

Copyright (c) 2015 by The American Association for Thoracic Surgery

http://dx.doi.org/10.1016/j.jtcvs.2014.12.045
} 